\title{
Analysis of the aerobic-anaerobic transition in elite cyclists during incremental exercise with the use of electromyography
}

\author{
Alejandro Lucía, Oscar Sánchez, Alfredo Carvajal, José L Chicharro
}

\begin{abstract}
Objectives-To investigate the validity and reliability of surface electromyography (EMG) as a new non-invasive determinant of the metabolic response to incremental exercise in elite cyclists. The relation between EMG activity and other more conventional methods for analysing the aerobic-anaerobic transition such as blood lactate measurements (lactate threshold (LT) and onset of blood lactate accumulation (OBLA)) and ventilatory parameters (ventilatory thresholds 1 and 2 $\left(V T_{1}\right.$ and $\left.V_{2} T_{2}\right)$ was studied.
\end{abstract}

Methods-Twenty eight elite road cyclists (age 24 (4) years; Vo $_{2}$ MAX 69.9 (6.4) $\mathrm{ml} / \mathrm{kg} / \mathrm{min}$; values mean (SD)) were selected as subjects. Each of them performed a ramp protocol (starting at $0 \mathrm{~W}$, with increases of $5 \mathrm{~W}$ every 12 seconds) on a cycle ergometer (validity study). In addition, 15 of them performed the same test twice (reliability study). During the tests, data on gas exchange and blood lactate levels were collected to determine $V_{1}, V_{1} T_{2}, L T$, and OBLA. The root mean squares of EMG signals (rms-EMG) were recorded from both the vastus lateralis and the rectus femoris at each intensity using surface electrodes.

Results-A two threshold response was detected in the rms-EMG recordings from both muscles in $90 \%$ of subjects, with two breakpoints, $\mathbf{E M G}_{\mathrm{T} 1}$ and $\mathrm{EMG}_{\mathrm{T} 2}$, at around $60-70 \%$ and $80-90 \%$ of $\dot{V}_{2} M A X$ respectively. The results of the reliability study showed no significant differences $(p>0.05)$ between mean values of $\mathrm{EMG}_{\mathrm{T} 1}$ and $\mathrm{EMG}_{\mathrm{T} 2}$ obtained in both tests. Furthermore, no significant differences $(p>0.05)$ existed between mean values of $\mathrm{EMG}_{\mathrm{T} 1}$, in the vastus lateralis and rectus femoris, and $V_{1}$ and LT (62.8 (14.5) and $69.0(6.2)$ and 64.6 (6.4) and $68.7(8.2) \%$ of $\dot{V}_{2} \mathrm{MAx}$ respectively), or between mean values of $\mathrm{EMG}_{\mathrm{T} 2}$, in the vastus lateralis and rectus femoris, and $\mathrm{VT}_{2}$ and OBLA (86.9 (9.0) and 88.0 (6.2) and $84.6(6.5)$ and 87.7 (6.4)\% of $\dot{\text { vo }}_{2} \mathrm{MAX}$ respectively).

Conclusion-rms-EMG may be a useful complementary non-invasive method for analysing the aerobic-anaerobic transition (ventilatory and lactate thresholds) in elite cyclists.

(Br J Sports Med 1999;33:178-185)

Keywords: electromyography; muscle; ventilatory threshold; lactate threshold; cycling; metabolic response
Surface electromyography (EMG) is an acceptable method for quantifying the total activity of working muscles and for estimating muscle fatigue non-invasively. ${ }^{1}$ An increase in EMG activity has been shown to reflect the recruitment of additional motor units and an increase in motor unit rate coding to compensate for the deficit in contractility resulting from impairment of fatigued motor units, as the strength of a muscle contraction increases. ${ }^{1}$ Along this line of thought, several studies have shown the existence of a non-linear increase in EMG during the aerobic-anaerobic transition phase in ergometer cycling. ${ }^{1-6}$ Indeed, an EMG threshold $\left(\mathrm{EMG}_{\mathrm{T}}\right)$ has been suggested to occur in the vastus lateralis, ${ }^{1-5}$ vastus medialis, ${ }^{35}$ rectus femoris, ${ }^{36-8}$ gastrocnemius, ${ }^{4}$ biceps femoris, ${ }^{6}$ and soleus ${ }^{6}$ of healthy not highly trained subjects during progressive tests on a cycle ergometer. The $\mathrm{EMG}_{\mathrm{T}}$, in turn, would represent the point where an increased contribution from fast twitch motor units occurs to maintain the required energy supply for muscle contraction. ${ }^{3}$ In other studies, in contrast, a linear relation has been reported between EMG and exercise intensity in ergometer cycling. ${ }^{9} 10$

In addition to these controversial findings, some questions still remain unanswered. First, only one study has assessed the test-retest reliability of the EMG response to progressive exercise. ${ }^{10}$ In this study, the EMG response was reported to be repeatable but linear (not threshold-like) during an incremental treadmill test. As reliability is an integral part of validity, ${ }^{11}$ no study has accurately determined the validity of the $\mathrm{EMG}_{\mathrm{T}}$ method for analysing the aerobicanaerobic transition. Furthermore, little research has been conducted using elite athletes (cyclists) as subjects, and the findings that have been reported seem somewhat controversial. ${ }^{78}$

The aim of this study was to investigate the validity and reliability of EMG as a new non-invasive determinant of the metabolic response to incremental exercise in elite cyclists. We studied the relation between EMG activity and other more conventional methods of analysing the aerobic-anaerobic transition, such as blood lactate measurements (lactate threshold (LT) and onset of blood lactate accumulation (OBLA)) and ventilatory parameters (ventilatory thresholds 1 and $2\left(\mathrm{VT}_{1}\right.$ and $\left.\mathrm{VT}_{2}\right)$ ).

Methods

SUBJECTS

Twenty eight elite male cyclists (age 24 (4) years; height $177.1(5.2) \mathrm{cm}$; body mass 67.2 (6.0) $\mathrm{kg}$; all values mean (SD)) participated in 
this study. Sixteen subjects were professional road cyclists with a minimum competition experience of three years, and some of them had won several professional races. The other 12 were elite road cyclists (competition experience at least two years in the amateur category). Written informed consent was given before participation in the experiments, in accordance with the institutional human subjects guidelines (Complutense University of Madrid).

STUDY PROTOCOL

Before each exercise testing session, subjects were familiarised with the equipment and procedures used in this investigation. In addition, they were previously instructed to refrain from intense training during the day before testing.

Fifteen subjects were randomly selected for the reliability study. Each of them performed two exercise tests on a bicycle ergometer (Ergometrics 900; Ergo-line, Barcelona, Spain) on different occasions and separated from each other by a period of no more than five days. Each of the two tests consisted of a ramp protocol until exhaustion, starting at $0 \mathrm{~W}$. The power output was increased by $5 \mathrm{~W}$ every 12 seconds and pedalling cadence was kept constant at $70-80 \mathrm{rpm}$. The selection of a ramp-like protocol instead of a graded steady state test was chosen for two reasons: $(a)$ in previous research conducted in our laboratory ${ }^{12}$ this type of protocol was used to analyse physiological responses - that is, ventilatory thresholds and lactate kinetics - in elite/ professional cyclists during incremental exercise; $(b)$ previous research showing a non-linear increase in the EMG response to incremental exercise used exercise protocols comparable with that used in our investigation. ${ }^{1-8}{ }^{13}$ For the validity study, each of 28 subjects performed a single bicycle ergometer test following the above protocol.

Exercise tests were terminated $(a)$ voluntarily by the subjects, $(b)$ when pedalling cadence could not be kept at least at $70 \mathrm{rpm}$, or (c) when established criteria of test termination were met. ${ }^{14}$ Each test was performed under similar conditions $\left(21-24^{\circ} \mathrm{C}\right.$ and $45-55 \%$ relative humidity).

ANALYSIS OF EXPIRED GAS AND DETERMINATION OF VENTILATORY THRESHOLD

During the tests, gas exchange data were collected continuously using an automated breath by breath system (CPX; Medical Graphics, St Paul, Minnesota, USA). The measuring instruments were calibrated before each test and the necessary environmental adjustments made. $\mathrm{VT}_{1}$ was determined using the criteria of an increase in the ventilatory equivalent for oxygen $\left(\dot{\mathrm{VE}} / \mathrm{V}_{\mathrm{O}_{2}}\right)$ with no increase in the ventilatory equivalent for carbon dioxide $\left(\dot{\mathrm{V} E} / \dot{\mathrm{V}} \mathrm{CO}_{2}\right)$ and the departure from linearity of $\dot{\mathrm{VE}}$, whereas $\mathrm{VT}_{2}$ was determined by using the criteria of an increase in both $\dot{\mathrm{VE}} / \dot{\mathrm{V}}_{2}$ and $\dot{\mathrm{VE}} / \mathrm{V}_{\mathrm{CO}_{2}} .{ }^{15}$ Two independent observers detected $\mathrm{VT}_{1}$ and $\mathrm{VT}_{2}$ following the criteria previously described. If they did not agree, the opinion of a third investigator was included.

ANALYSIS OF BLOOD LACTATE

Blood samples $(25 \mu \mathrm{l})$ for the measurement of blood lactate (YSI 23L; Yellow Springs Instruments, Yellow Springs, Ohio, USA) were taken from fingertips at rest, every two minutes during the test, and immediately after termination of exercise.

LT was determined by examining the "lactate concentration-power output (W)" relationship during the tests, using the methodology previously described by Weltman and co-workers. ${ }^{16}$ Thus the greatest power output that was not associated with a rise in lactate concentration above baseline was designated as the power output corresponding to LT. This always occurred just before the curvilinear increase in blood lactate observed with subsequent exercise intensities. A lactate increase of at least $0.2 \mathrm{mM}$ (the error associated with the lactate analyser) was required for LT determination. OBLA, on the other hand, was defined as the power output corresponding to a blood lactate concentration of $4.0 \mathrm{mmol} / \mathrm{l}^{17}$

EMG

Electrode placement

Surface EMG recordings were taken from the vastus lateralis and rectus femoris (at sites respectively approximately one third and one half of the perpendicular distance from the superior border of the patella to the greater trochanter). Pairs of surface electrodes (Bluesensor Medicotest $\mathrm{Ag} / \mathrm{AgCl}$ electrodes; Rugmarken, Denmark) were attached to the skin with a $4 \mathrm{~cm}$ interelectrode distance. The electrodes were placed longitudinally with respect to the underlying muscle fibre arrangement. For those subjects who performed the test twice (reliability study), the skin was tattoed using ink in order to place the electrodes on the same site on the two tests. Two reference electrodes were placed over the anterior superior spine of the iliac crest. Before electrode application, the skin was shaved and abrased using sandpaper and cleaned with alcohol to minimise the source impedance. A saline EMG electrode gel was placed between the electrode and the underlying skin to enhance signal conductivity. The cables connected to the electrodes to measure myoelectrical activity were firmly attached with tape to minimise artefacts from leg movements.

\section{EMG instrumentation and procedures}

Myoelectrical activity was recorded using a ME3000P analyser (ME3000P; Mega Electronics Ltd, Kuopio, Finland). The measurement sensitivity of the instrument is $\pm 1 \mu \mathrm{V}$ and its range for bipolar EMG signals is $\pm 5000 \mu \mathrm{V}$. The raw EMG signals were band-pass filtered between 20 and $480 \mathrm{~Hz}$, amplified, and converted from analogue to digital at a sampling rate of $1 \mathrm{kHz}$. An EMG power spectral density was then computed for two second sampling periods, at fixed intervals throughout the tests, and the root mean square voltage (rms-EMG) contained in each two second 
spectrum was calculated (in $\mu \mathrm{V}$ ). ${ }^{18}$ The rmsEMG was used as an indication of the "total myoelectric activity" of an exercising muscle, as it has been previously shown that this computation is (a) an accurate measure of EMG amplitude and $(b)$ is highly correlated with the number of active motor units (fibre recruitment)..$^{18} 19$

\section{Determination of EMG breakpoint}

Previous pilot studies conducted in our laboratory suggested the existence of a two threshold response in elite cyclists, with a secong EMG breakpoint occurring at near to maximum intensities, which is easy to detect visually. To establish objective criteria for the determination of one or two breakpoints in the EMG power output response, we used a computer algorithm (Centro de Proceso de Datos, Complutense University of Madrid) that models rms-EMG response to exercise using multisegment linear regression.

With this method, a single linear regression is initially fitted to all data points and is used for later statistical comparisons. A brute force method is then used to fit two lines to the data points. The program calculates regression lines for all possible divisions of the data into two contiguous groups, and the pair of lines yielding the least pooled residual sum of squares is chosen as representing the best fit. The intersection point between these two lines occurred near the end of the test in all the subjects.

Thereafter the program attempts to fit a third line to the data in order to detect another breakpoint in the EMG data. The third middle segment is obtained by methodically adding points on the left side of the two line regression intersection point. The new regression line is then calculated and extended in the direction that yielded the lower sum of squares.

Finally, an analysis of variance determines whether a significant $(\mathrm{p}<0.05)$ reduction in the total sum of squares is achieved by the addition of a third line segment. The first $\left(\mathrm{EMG}_{\mathrm{T} 1}\right)$ and second $\left(\mathrm{EMG}_{\mathrm{T} 2}\right)$ EMG thresholds are then reported as the first and second intersection points respectively of the computerised model.

COMPARISONS BETWEEN VT $1, \mathrm{VT}_{2}, \mathrm{LT}$, OBLA, AND $\mathrm{EMG}_{\mathrm{T} 1} \mathrm{AND}_{\mathrm{EMG}} \mathrm{T} 2$

Each individual value of $\mathrm{VT}_{1}, \mathrm{VT}_{2}, \mathrm{LT}, \mathrm{OBLA}$, and $\mathrm{EMG}{ }_{\mathrm{T} 1}$ and $\mathrm{EMG}_{\mathrm{T} 2}$ corresponded to a certain time point during the tests, which in turn elicited a certain value of $\dot{\mathrm{V}}_{2}$ and power output (W). Therefore, in order to compare the exercise intensity at which all thresholds occurred, mean values of $\mathrm{VT}_{1}, \mathrm{VT}_{2}$, LT, OBLA, and $\mathrm{EMG} \mathrm{T}_{\mathrm{T} 1}$ and $\mathrm{EMG}_{\mathrm{T} 2}$ were expressed in both $\dot{\mathrm{VO}}_{2}(\mathrm{ml} / \mathrm{kg} / \mathrm{min})$ and $\%$ of $\dot{\mathrm{V}}_{2} \mathrm{MAX}$, and in power output (W).

DATA ANALYSIS

In the group of 15 subjects that performed the test on two different days (reliability study), a paired Student's $t$ test was used to compare mean values of $\mathrm{EMG}_{\mathrm{T} 1}$ and $\mathrm{EMG}_{\mathrm{T} 2}$ obtained with both tests. Intraclass Pearson's correlation coefficients were also calculated to determine the degree of correlation between mean values of $\mathrm{EMG}_{\mathrm{T} 1}$ and $\mathrm{EMG}_{\mathrm{T} 2}$ reported with repeated tests.

In all 28 subjects (validity study), mean values of $\mathrm{VT}_{1}$, LT (criterion methods), and $\mathrm{EMG}_{\mathrm{T} 1}$, on one hand, and those of $\mathrm{VT}_{2}$, OBLA (criterion methods), and $\mathrm{EMG}_{\mathrm{T} 2}$, on the other, were compared by using a one way repeated measures analysis of variance. In addition, total errors $\left(\sum\left(\mathrm{Y}-\mathrm{Y}^{\prime}\right)^{2} / \mathrm{N}^{1 / 2}\right.$ where $\mathrm{Y}$ is the criterion value and Y' the predicted value) and Pearson's correlation coefficients (criterion $v$ predicted) were calculated to examine the error inherent in the EMG prediction technique. Further analysis of validity of the EMG method was accomplished by applying the procedures suggested by Bland and Altman. ${ }^{20}$ For this analysis, the mean differences (bias) and standard deviation (SD) of the differences between the mean values (in W) obtained with the two methods (EMG $v$ criterion methods) were calculated. The data are presented graphically comparing the difference between the methods against their average value in W. The mean difference (bias) plus and minus two standard deviations is indicated on the graph. In this way, the bias and precision of the EMG technique could be calculated.

Significance was set at $\mathrm{p}<0.05$ for all statistical analyses.

\section{Results}

MAXIMAL VALUES

Maximal values of $\dot{\mathrm{V}}_{2}$ and power output averaged $69.9(6.4) \mathrm{ml} / \mathrm{kg} / \mathrm{min}$ (range $60.0-82.3$ ) and 432.8 (49.1) W (range 364-518) at the end of exercise.

PATTERN OF EMG RESPONSE

A two threshold response was detected in $90 \%$ of subjects in both vastus lateralis and rectus femoris, and the two breakpoints $\mathrm{EMG}_{\mathrm{T} 1}$ and $\mathrm{EMG}_{\mathrm{T} 2}$ occurred around $60-70 \%$ and $80-90 \%$ of $\dot{\mathrm{V}}_{2} \mathrm{MAX}$. In $10 \%$ of the cases, $\mathrm{EMG}_{\mathrm{T} 1}$ could not be detected in either of the two muscles studied, whereas $\mathrm{EMG}_{\mathrm{T} 2}$ was found in all 28 subjects.

Figure 1 shows an example of an EMG response in one subject.

\section{RELIABILITY}

No significant differences existed between mean values of either $\mathrm{EMG}_{\mathrm{T} 1}$ or $\mathrm{EMG}_{\mathrm{T} 2}$ obtained in both tests $(\mathrm{p}<0.05)$ (table 1$)$.

Intraclass correlation coefficients $(r)$ between repeated measurements were significant $(\mathrm{p}<0.05)$ and high (table 1$).$

\section{VALIDITY}

Comparison between $V T_{1}, L T$, and $E M G_{T 1}$ Using the methodologies described above, $\mathrm{VT}_{1}$ and LT could be detected in $100 \%$ of subjects. Average values of LT occurred at a blood lactate concentration of $1.9(0.5) \mathrm{mM}$. Table 2 presents mean values of $\mathrm{VT}_{1}, \mathrm{LT}$, and $\mathrm{EMG}_{\mathrm{T} 1}$ expressed in $\dot{\mathrm{V}}_{2}(\mathrm{ml} / \mathrm{kg} / \mathrm{min}), \%$ of $\dot{\mathrm{V}}_{2} \mathrm{MAX}$, and W. No significant differences $(p>0.05)$ were found between means. 


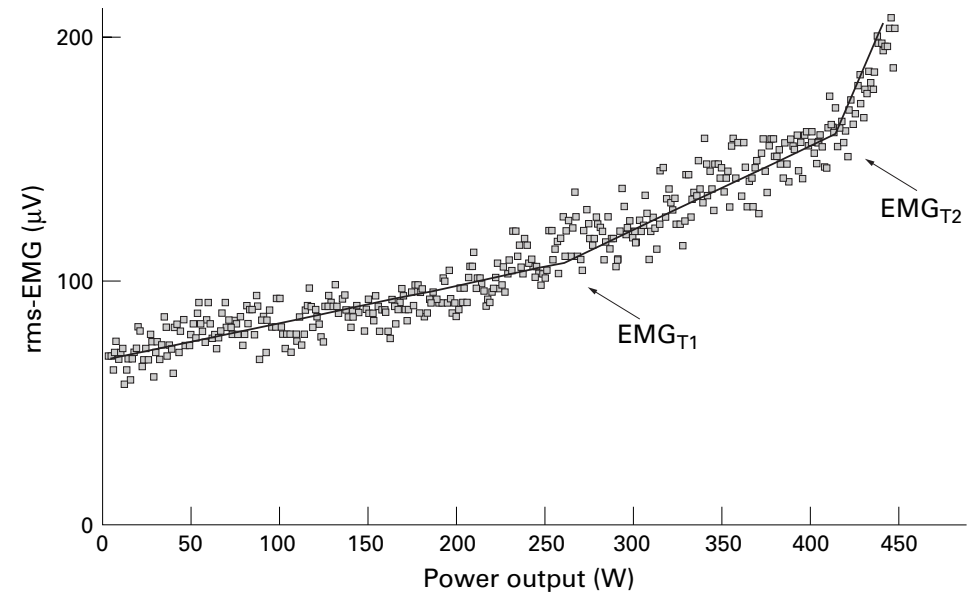

Figure 1 Example of electromyographic (EMG) recording in one subject. Each data point represents root mean square of an EMG signal (rms-EMG), recorded at two second intervals. The rms-EMG data against time were fitted mathematically to the corresponding straight lines by computerised multisegment linear regression. $E M G_{T 1}$ and $E M G_{T 2}$ are first and second EMG thresholds.

Table 1 Reliability of measurements of the electromyographic thresholds $E M G_{T 1}$ and $E M G_{T 2}$

\begin{tabular}{|c|c|c|c|}
\hline & First test & Second test & Correlation (r) \\
\hline \multicolumn{4}{|l|}{ Vastus lateralis } \\
\hline \multicolumn{4}{|l|}{$\mathrm{EMG}_{\mathrm{T} 1}$} \\
\hline$\dot{\mathrm{V}}_{2}(\mathrm{ml} / \mathrm{kg} / \mathrm{min})$ & $45.5(2.5)$ & $43.9(3.0)$ & 0.76 \\
\hline$\% \dot{\mathrm{V}}_{2} \mathrm{MAX}$ & $68.8(4.0)$ & $63.3(4.2)$ & 0.82 \\
\hline W & 252.7 (17.6) & $238.2(17.9)$ & 0.83 \\
\hline \multicolumn{4}{|l|}{$\mathrm{EMG}_{\mathrm{T} 2}$} \\
\hline $\mathrm{VO}_{2}(\mathrm{ml} / \mathrm{kg} / \mathrm{min})$ & $59.7(1.4)$ & $62.6(1.4)$ & 0.96 \\
\hline$\% \dot{\mathrm{V}}_{2} \mathrm{MAX}$ & $90.2(1.3)$ & $90.2(2.3)$ & 0.87 \\
\hline W & $367.6(15.4)$ & $372.6(14.7)$ & 0.86 \\
\hline \multirow{2}{*}{\multicolumn{4}{|c|}{$\begin{array}{l}\text { Rectus femoris } \\
\text { EMG }_{\mathrm{TI}}\end{array}$}} \\
\hline $\mathrm{EMG}_{\mathrm{T} 1}$ & & & \\
\hline$\dot{\mathrm{V}}_{\mathrm{O}_{2}}(\mathrm{~m} / \mathrm{kg} / \mathrm{min})$ & $48.7(2.5)$ & $47.3(1.7)$ & 0.89 \\
\hline$\% \mathrm{VO}_{2} \mathrm{MAX}$ & $70.4(3.0)$ & $68.4(2.9)$ & 0.85 \\
\hline & $280.2(22.4)$ & $268.2(20.8)$ & 0.96 \\
\hline \multicolumn{4}{|l|}{$\mathrm{EMG}_{\mathrm{T} 2}$} \\
\hline $\mathrm{VO}_{2}(\mathrm{ml} / \mathrm{kg} / \mathrm{min})$ & $59.6(2.1)$ & $59.8(1.5)$ & 0.73 \\
\hline$\% \mathrm{VO}_{2} \mathrm{MAX}$ & $89.9(2.0)$ & $86.2(1.8)$ & 0.75 \\
\hline $\mathrm{W}$ & $374.2(20.7)$ & $360.8(20.0)$ & 0.87 \\
\hline
\end{tabular}

All values are expressed as mean (SEM).

No significant differences existed between means $(\mathrm{p}>0.05)$. All correlation coefficients were significant $(\mathrm{p}<0.05)$.

Table 2 Comparison between mean values of the first electromyographic threshold $\left(E M G_{T 1}\right)$, the first ventilatory threshold $\left(V T_{1}\right)$, and the lactate threshold $(L T)$

\begin{tabular}{|c|c|c|c|c|}
\hline & \multicolumn{2}{|l|}{$E M G_{T I}$} & \multirow[b]{2}{*}{$V T_{1}$} & \multirow[b]{2}{*}{$L T$} \\
\hline & Vastus lateralis & Rectus femoris & & \\
\hline $\begin{array}{l}\dot{\mathrm{V}}_{2}(\mathrm{ml} / \mathrm{kg} / \mathrm{min}) \\
\% \dot{\mathrm{V}}_{2} \mathrm{MAX} \\
\mathrm{W}\end{array}$ & $\begin{array}{r}43.9(1.7) \\
62.8(2.8) \\
240.3(9.8)\end{array}$ & $\begin{array}{c}47.0(1.5) \\
69.0(2.1) \\
270.8(13.8)\end{array}$ & $\begin{array}{c}45.1(1.2) \\
64.6(1.1) \\
257.8(10.0)\end{array}$ & $\begin{array}{c}46.1(1.6) \\
68.7(1.7) \\
269.8(12.9)\end{array}$ \\
\hline
\end{tabular}

All values are expressed as mean (SEM).

No significant differences existed between means ( $p>0.05)$.

Table 3 Comparison between mean values of the second electromyographic threshold $\left(E M G_{T 2}\right)$, the second ventilatory threshold $\left(V T_{2}\right)$, and the onset of blood lactate accumulation $(O B L A)$

\begin{tabular}{lcccc}
\hline \multicolumn{3}{c}{$E M G_{T 2}$} & & \\
\cline { 2 - 3 } & Vastus lateralis & Rectus femoris & $V T_{2}$ & \multirow{2}{*}{ OBLA } \\
\hline$\dot{\mathrm{VO}}_{2}(\mathrm{ml} / \mathrm{kg} / \mathrm{min})$ & $61.5(1.1)$ & $59.7(1.3)$ & $59.0(1.1)$ & $60.7(1.5)$ \\
$\% \mathrm{VO}_{2} \mathrm{MAX}$ & $86.9(1.5)$ & $88.0(1.4)$ & $84.6(6.5)$ & $87.7(1.3)$ \\
$\mathrm{W}$ & $371.1(9.2)$ & $367.5(14.1)$ & $352.8(11.4)$ & $377.6(13.0)$
\end{tabular}

All values are expressed as mean (SEM)

No significant differences existed between means $(p>0.05)$.

Comparison between $V T_{2}, O B L A$, and $E M G_{T 2}$ Using the methodologies described above, $\mathrm{VT}_{2}$ and OBLA could be detected in $100 \%$ of subjects. Table 3 presents mean values of $\mathrm{VT}_{2}$ and
Table 4 Total errors

\begin{tabular}{|c|c|c|}
\hline & $\begin{array}{l}\text { Vastus } \\
\text { lateralis }\end{array}$ & $\begin{array}{l}\text { Rectus } \\
\text { femoris }\end{array}$ \\
\hline \multicolumn{3}{|l|}{$\mathrm{EMG}_{\mathrm{T} 1} v \mathrm{VT}_{1}$} \\
\hline$\dot{\mathrm{VO}}_{2}$ & 2.1 & 1.9 \\
\hline$\% \dot{\mathrm{V}}_{2} \operatorname{MAX}$ & 3.0 & 2.4 \\
\hline $\mathrm{W}$ & 14.1 & 16.7 \\
\hline \multicolumn{3}{|l|}{$\mathrm{EMG}_{\mathrm{T} 1} v \mathrm{LT}$} \\
\hline$\dot{\mathrm{VO}}_{2}$ & 2.3 & 2.2 \\
\hline$\% \dot{\mathrm{V}}_{2} \operatorname{MAX}$ & 2.0 & 2.7 \\
\hline $\mathrm{W}$ & 16.3 & 19.0 \\
\hline \multicolumn{3}{|l|}{$\mathrm{EMG}_{\mathrm{T} 2} v \mathrm{VT}_{2}$} \\
\hline$\dot{\mathrm{V}} \mathrm{O}_{2}$ & 1.6 & 1.7 \\
\hline$\% \dot{\mathrm{V}}_{2} \operatorname{MAX}$ & 1.9 & 1.8 \\
\hline $\mathrm{W}$ & 14.8 & 18.0 \\
\hline \multicolumn{3}{|c|}{$\mathrm{EMG}_{\mathrm{T} 2} v$ OBLA } \\
\hline$\dot{\mathrm{V}} \mathrm{O}_{2}$ & 1.8 & 2.0 \\
\hline$\% \dot{\mathrm{VO}}_{2} \mathrm{MAX}$ & 2.0 & 1.9 \\
\hline W & 16.0 & 19.3 \\
\hline
\end{tabular}

$\mathrm{EMG}_{\mathrm{T} 1}$ and $\mathrm{EMG}_{\mathrm{T} 2}$, first and second electromyographic thresholds; $\mathrm{VT}_{1}$ and $\mathrm{VT}_{2}$, ventilatory thresholds 1 and 2; LT, lactate threshold; OBLA, onset of blood lactate accumulation.

Table 5 Significant correlation coefficients $(p<0.05)$

\begin{tabular}{lll} 
& $E M G_{T 1}$ & $E M G_{T 2}$ \\
\hline $\mathrm{VT}_{1}$ & 0.66 & - \\
$\mathrm{VT}_{2}$ & - & 0.82 \\
$\mathrm{LT}$ & 0.64 & 0.80 \\
OBLA & - &
\end{tabular}

$\mathrm{EMG}_{\mathrm{T} 1}$ and $\mathrm{EMG}_{\mathrm{T} 2}$, first and second electromyographic thresholds; $\mathrm{VT}_{1}$ and $\mathrm{VT}_{2}$, ventilatory thresholds 1 and 2; LT, lactate threshold; OBLA, onset of blood lactate accumulation.

$\mathrm{EMG}_{\mathrm{T} 2}$ expressed in $\dot{\mathrm{V}}_{2}(\mathrm{ml} / \mathrm{kg} / \mathrm{min}), \%$ of $\dot{\mathrm{V}}_{\mathrm{O}_{2} \mathrm{MAX}}$, and W. No significant differences ( $p>0.05)$ existed between means.

\section{Total errors}

Table 4 gives total errors.

Correlations

Table 5 gives significant correlations between the measured variables.

\section{Bland and Altman procedure}

The statistical approach reported by Bland and Altman $^{20}$ was applied to assess the degree of agreement between the following methods: $\mathrm{EMG}_{\mathrm{T} 1}$ (vastus lateralis) $v \mathrm{VT}_{1}$ and LT (figs 2 and 3 respectively); $\mathrm{EMG}_{\mathrm{T} 2}$ (vastus lateralis) $v$ $\mathrm{VT}_{2}$ and OBLA (figs 4 and 5 respectively). In general, the bias and precision of the EMG method were relatively low, especially for $\mathrm{EMG}_{\mathrm{T} 2}$. In addition, for all comparisons, at least $90 \%$ of the individual values were within the limits of agreement.

\section{Discussion}

To our knowledge, this is the first report to determine the validity of the EMG method for analysing the aerobic-anaerobic transition phase in top level athletes (professional cyclists) during cycle ergometry using a ramp test. Previous studies on EMG response during such protocols have been conducted with subjects of considerably lower fitness levels (in most studies, mean $\dot{\mathrm{V}}_{2} \mathrm{MAX}<65 \mathrm{ml} / \mathrm{kg} / \mathrm{min}$, and in all of them mean peak power output $<360 \mathrm{~W})$ than those selected for our investigation $\left(\dot{\mathrm{VO}}_{2} \operatorname{MAX} 69.9(6.4) \mathrm{ml} / \mathrm{kg} / \mathrm{min}\right.$; peak power output $432.8(49.1) \mathrm{W})$. To date, no conclusion could be inferred from previous research 


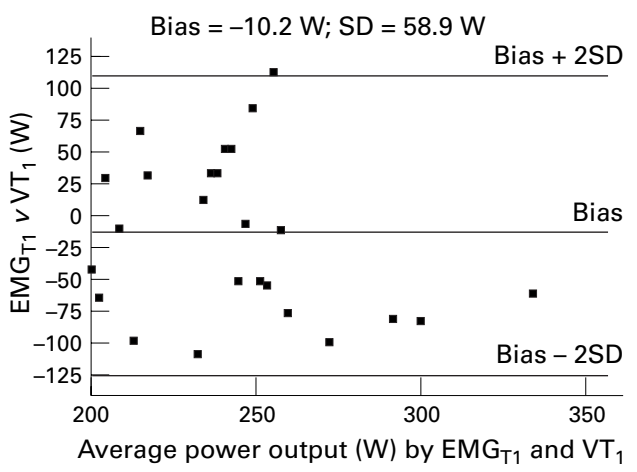

Figure 2 Graphic analysis of power output $(W)$ data corresponding to the first electromyographic threshold $\left(E M G_{T 1}\right.$; vastus lateralis) and ventilatory threshold 1 (VT,.

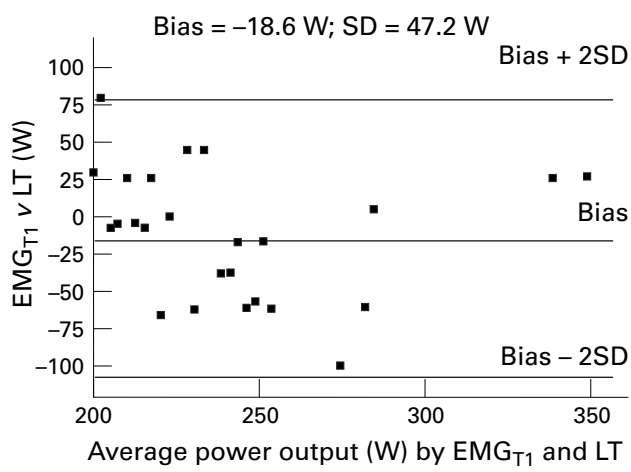

Figure 3 Graphic analysis of power output (W) data corresponding to the first electromyographic threshold $\left(E M G_{T 1}\right.$; vastus lateralis) and lactate threshold (LT).

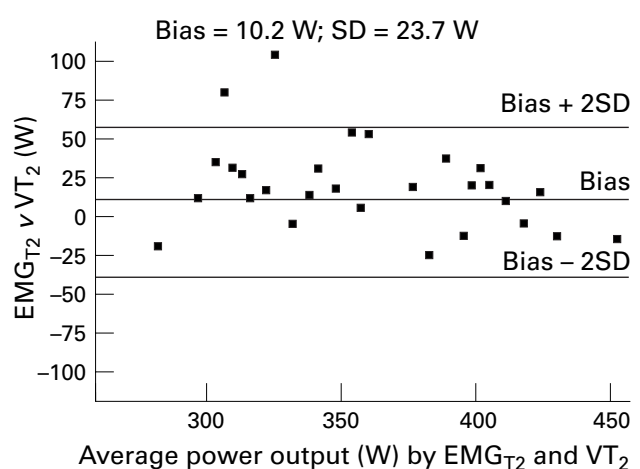

Figure 4 Graphic analysis of power output (W) data corresponding to the second electromyographic threshold (EMG $G_{T 2}$; vastus lateralis) and the ventilatory threshold 2 $\left(V T_{2}\right)$

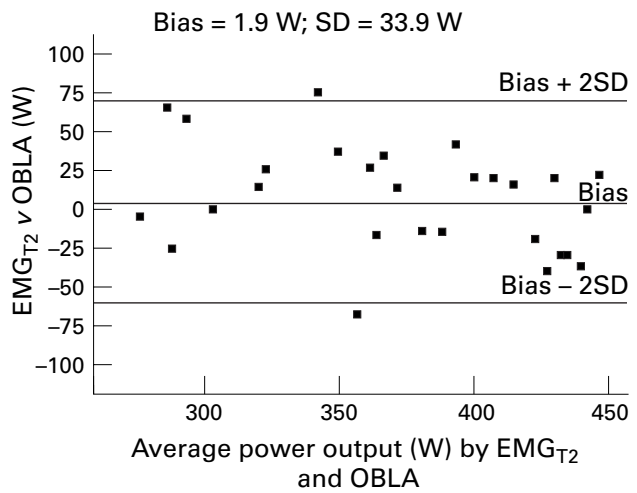

Figure 5 Graphic analysis of power output (W) data corresponding to the second electromyographic threshold (EMG $G_{T 2}$; vastus lateralis) and the onset of blood lactate accumulation $(O B L A)$. on the EMG response of elite cyclists reaching high power outputs during exercise testing. In addition, no previous study has assessed the reliability of the EMG method during a ramp protocol. As reliability is an integral part of validity, ${ }^{11}$ no previous report has assessed the validity of such a method for analysing the aerobic-anaerobic transition.

A novel finding of our study was that in elite cyclists the EMG activity of the muscles primarily involved in pedalling (vastus lateralis, rectus femoris) show a two threshold response with two distinct breakpoints, $\mathrm{EMG}_{\mathrm{T} 1}$ and $\mathrm{EMG}_{\mathrm{T} 2}$, occurring at an exercise intensity of $60-70 \%$ and $80-90 \%$ of $\dot{\mathrm{V}}_{2} \mathrm{MAX}$ respectively. A second finding of our investigation was that the EMG method is both valid and reliable for analysing the aerobic-anaerobic transition during exercise. Finally, in elite cyclists it appears that muscle fatigability is not significantly affected by muscle fibre type distribution, as $\mathrm{EMG}_{\mathrm{T} 1}$ and $\mathrm{EMG}_{\mathrm{T} 2}$ occurred at similar intensities in the vastus lateralis and rectus femoris.

METHODOLOGICAL LIMITATIONS

Our study is not without potential limitations. Firstly, the literature on the usefulness of EMG in the determination of ventilatory or lactate thresholds is somewhat controversial. The differences in results could be attributed to difficulties in reliably following the myoelectrical activities in working muscles. ${ }^{4}$ In addition, during exertion the skin blood flow and temperature increase. ${ }^{21}$ This phenomenon could induce alterations in the electrical properties of the skin and affect the records of surface EMG. In this regard, previous research has shown no correlation between skin temperature and EMG measurements, excluding the possibility that EMG breakpoints are related to changes in electrical properties of the skin. ${ }^{4}$

On the other hand, although we measured the myoelectrical activity of the muscles (vastus lateralis and rectus femoris) involved during the work (descending) period of pedalling only, no records were obtained for other musclesfor example, gastrocnemius, tibialis anteriorthat are also involved during the rest (ascending) period of pedalling exercise. However, previous research has shown that a non-linear increase in EMG also occurs in the gastrocnemius muscle. ${ }^{4}$ In addition, previous $\mathrm{EMG}^{22}$ and biopsy studies ${ }^{23}$ suggest that the muscle most heavily involved during cycling is the vastus lateralis.

Furthermore, a single ramp increment $(5 \mathrm{~W}$ every 12 seconds) was chosen in our protocol, whereas the results of a previous study using different ramp slopes (10, 20, 30 and 40 $\mathrm{W} / \mathrm{min}$ ) suggest that the metabolic state at which the $\mathrm{EMG}_{\mathrm{T}}$ occurs in untrained subjects may differ during the different ramp exercises. ${ }^{24}$ Further research should be conducted with elite cyclists to determine the influence of varying ramp protocols on the EMG response.

Finally, the results obtained during a progressive test in a cycle ergometer using a fixed cadence (70-80 rpm), as was used in this investigation, cannot be easily extrapolated to 
real sports situations (in contrast with more specific field tests). A similar study should be conducted under field conditions with unfixed cadence, to corroborate the implications of our findings. In this regard, it could be hypothesised that the pattern of muscle fibre recruitment may differ in laboratory to outfield conditions. Indeed, in laboratory tests using a fixed cadence the exercise intensity is augmented solely by increasing the force applied to each pedal stroke, ${ }^{25}$ whereas, during physical activity (cycling, running, swimming, etc) under real conditions, both the cadence and the force applied during each movement are involved in the increase in exercise intensity. ${ }^{19}$ Therefore, with increasing work intensity, the muscular power required in tests performed at a fixed cadence is considerably higher than that required by tests at increasing cadence-that is, field tests-and under actual cycling conditions. $^{25}$

COMPARISON WITH PREVIOUS STUDIES

Previous studies with healthy non-elite subjects have shown the occurrence of a single point (the "EMG ${ }_{\mathrm{T}}$ ") at which the increase in EMG of quadriceps muscles becomes non-linear during exercise protocols comparable with that used in our investigation. ${ }^{1-824}$ Furthermore, a similar response has been reported in cardiac transplant patients in a study conducted in our laboratory. ${ }^{13}$ This $\mathrm{EMG}_{\mathrm{T}}$, in turn, has been shown to occur during the transition from aerobic to anaerobic metabolism, at about $65-70 \%$ of $\dot{\mathrm{V}}_{2} \mathrm{MAX}$ in healthy subjects ${ }^{7-9} 12$ and about $60 \%$ of $\dot{\mathrm{V}}_{2} \mathrm{MAX}$ in cardiac transplant patients. ${ }^{13}$ In these studies, the aerobicanaerobic transition was expressed using ventilatory parameters $\left(\mathrm{VT}_{1}\right)^{2-413}$ or lactate measurements based on a first ${ }^{2313}$ or second ${ }^{45}$ abrupt increase in blood lactate (corresponding to blood lactate concentrations of about 2 and $4 \mathrm{mM}$ respectively). Indeed, the $\mathrm{EMG}_{\mathrm{T}}$ may occur as a result of a change in the pattern of motor unit recruitment from predominantly slow twitch motor units to fast twitch motor units, which could contribute to the accumulation of circulating lactate during exercise. ${ }^{2} 324$ The $\mathrm{EMG}_{\mathrm{T}}$ has therefore been suggested as an alternative non-invasive method for determination of ventilatory and/or lactate thresholds.

Our results are in overall agreement with those of previous studies, as no significant differences were observed between the exercise intensity corresponding to the $\mathrm{EMG}_{\mathrm{T} 1}$ and that corresponding to $\mathrm{VT}_{1}$ or $\mathrm{LT}$. It follows that rms-EMG may represent a complementary non-invasive indicator of the aerobic-anaerobic transition during gradual exercise tests. A second breakpoint $\left(\mathrm{EMG}_{\mathrm{T} 2}\right)$, however, also occurred in our subjects, which needs further discussion.

\section{COINCIDENCE OF EMG AND BLOOD LACTATE} RESPONSES

The reason for an abrupt increase in EMG activity above a certain exercise intensity is not fully understood, and could be at both a local (muscle fatigue) and a generalised level. With respect to the first phenomenon, it can be assumed that after LT is reached, ATP supply from slow twitch oxidative (type I) fibres through oxidative phosphorylation becomes inadequate and therefore must be supplemented by using energy reserves available through anaerobic glycolysis, leading to metabolic acidosis. Accumulation of hydrogen ions, in turn, has been shown to impair excitationcontraction coupling through impairment of the function of (a) the sodium/potassium ATPase of the sarcolemma, (b) the calcium ATPase of the sarcoplasmic reticulum, or (c) the myosin ATPase involved in actin-myosin interaction. ${ }^{19}$ Moreover, high lactate levels per se may disrupt excitation-contraction coupling. ${ }^{26}$ Thus, under these physiological conditions, in order to compensate for the deficit in contractility resulting from impairment of fatigued motor units, muscle force output must be increased during an incremental exercise test through recruitment of additional motor units, particularly those made of fast twitch oxidative glycolytic (type IIa) and fast twitch glycolytic (type IIb) muscle fibres. The observed non-linear increases in rms-EMG $\left(\mathrm{EMG}_{\mathrm{T} 1}\right.$ and $\left.\mathrm{EMG}_{\mathrm{T} 2}\right)$ could therefore be explained by a progressive recruitment of motor units with possible participation of type IIa and IIb fibres (at the $\mathrm{EMG}_{\mathrm{T} 1}$ and $\mathrm{EMG}_{\mathrm{T} 2}$ respectively) producing larger action potentials, followed by some degree of synchronisation of motor unit potentials as these fibres may undergo progressive fatigue. ${ }^{2}$ In fact, our findings are in agreement with previous research with muscle biopsy samples, which has shown a 1:1 relation between the fraction of active fibres in the vastus lateralis and the intensity (determined as a percentage of $\dot{\mathrm{VO}}_{2} \mathrm{MAX}$ ) of cycle ergometer exercise following this pattern: at about $40 \%$ of $\dot{\mathrm{V}}_{2} \mathrm{MAX}$, almost only type I fibres are recruited, whereas at about $60 \%$ of $\dot{\mathrm{V}}_{\mathrm{O}_{2} \mathrm{MAX}}$ $\left(\mathrm{EMG}_{\mathrm{T} 2}\right)$ both type I and IIa are activated, and during severe exercise (about $90 \%$ of $\dot{\mathrm{VO}}_{2} \mathrm{MAX}$ or $\mathrm{EMG}_{\mathrm{T} 2}$ ), fibres of type I, IIa, and IIb are recruited. ${ }^{27}$

However, the underlying mechanism may not be limited to a local level (muscle fatigue induced by metabolic acidosis). Such an hypothesis is supported by previous research showing that changes in EMG activity consistent with motor unit recruitment and muscle fatigue can also be recorded from patients with McArdle's syndrome ${ }^{28}$ or from normal subjects under conditions of unchanging muscle $\mathrm{pH} .{ }^{29}$ Therefore, other ions-for example, potassium, ammonia, adenosine monophosphate, and magnesium - may be responsible for altering muscle function during exercise. ${ }^{18}$ In addition, Airaksinen and co-workers ${ }^{4}$ showed that both working (vastus lateralis and gastrocnemius) and non-working (frontalis) muscles showed a shift in EMG at the same load, suggesting that the breakpoint(s) observed in muscle electrical activity may not only be attributed to peripheral fatigue. Thus the explanation could also be a change in the basic activation of muscles in general. Such a generalised response could be due to a change in the membrane function initiated not only by an 
increase in lactate levels but also by other factors such as an increase in neural or hormonal activity. ${ }^{4}$

\section{COINCIDENCE OF EMG AND VENTILATORY}

RESPONSES

The correlation encountered between $\mathrm{VT}_{1}$ and $\mathrm{EMG}_{\mathrm{T} 1}$, on the one hand, and $\mathrm{VT}_{2}$ and $\mathrm{EMG}_{\mathrm{T} 2}$, on the other, could be due to a muscle derived signal to ventilation. Indeed, previous studies provide evidence for the existence of ergoreceptors or mechanoreceptors which respond to increases in the work performed per unit muscle. $^{30}$ Moreover, Morikawa and co-workers ${ }^{31}$ found that a ventilatory response occurred in normal subjects with both active and passive leg exercise, but was absent with passive exercise in patients with thoracic spine transection. It could then be hypothesised that ventilation shows a first deflection point $\left(\mathrm{VT}_{1}\right)$ when muscle work is increased (at an exercise intensity corresponding to $\mathrm{EMG}_{\mathrm{T} 1}$ ) and a further increase $\left(\mathrm{VT}_{2}\right)$ when additional motor units are recruited to maintain power outputthat is, recruitment of type IIb fibres at the $\mathrm{EMG}_{\mathrm{T} 2}$.

On the other hand, the ventilatory thresholds observed during an incremental test could be elicited by enhancement of the neural activity that originates directly from the subthalamic motor region or indirectly via $\alpha-\gamma$ coactivation of motoneurons innervating the muscle fibres of exercising limbs. ${ }^{32}$ This increase in neural activity may occur during incremental exercise in response to the need to progressively recruit additional motor units comprised of type IIa (possibly at the $\mathrm{EMG}_{\mathrm{T} 1}$ ) and IIb fibres (possibly at the $\mathrm{EMG}_{\mathrm{T} 2}$ ) respectively as the work rate is increased and individual fibres begin to fatigue. ${ }^{32}$ Indeed, numerous investigations have shown simultaneous increases in ventilation and EMG activity during incremental exercise. ${ }^{3413} 32$

The results of a recent study, however, do not support a link between motor unit recruitment and ventilation, as evidenced by the disassociation between the $\mathrm{EMG}_{\mathrm{T}}$ of the rectus femoris and the ventilatory threshold that existed in trained cyclists after glycogen depletion. ${ }^{8}$ A similar study should be conducted with professional cyclists in order to assess the effects of glycogen depletion on the rms-EMG response.

ABSENCE OF EMG $\mathrm{T}_{\mathrm{T} 2}$ IN PREVIOUS RESEARCH The question remains to be answered of why a second EMG threshold $\left(\mathrm{EMG}_{\mathrm{T} 2}\right)$ was found only in this study, in contrast with previous research. In this regard, a possible explanation could be that the significant increases in neural activity to the exercising muscles that occur at $\mathrm{EMG}_{\mathrm{T} 2}$ were not detected in previous studies because EMG activity was mainly recorded from a single muscle - that is, the vastus lateralis. This hypothesis is supported by the findings of Green and Patla, ${ }^{19}$ which showed that many different muscle groups contribute to the completion of an incremental exercise task and that the average level of activation increases differentially among the muscle groups. It may be possible that only highly trained cyclists (such as those selected for our study) are able to effectively recruit a sufficient number of motor units (especially those with fast fibres) within individual muscles (vastus lateralis or rectus femoris) at near to maximum intensities during an incremental test such as to induce a second breakpoint in the EMG response to exercise. Indeed, $\mathrm{EMG}_{\mathrm{T} 2}$ occurred at a power output as high as $370 \mathrm{~W}$, which in turn elicited a $\mathrm{V}_{\mathrm{O}_{2}}$ of about $60 \mathrm{ml} / \mathrm{kg} / \mathrm{min}$. Both values are well above the maximal levels of power output and $\dot{\mathrm{V}}_{2}$ reported in most previous EMG studies $(<350$ W and $<55 \mathrm{ml} / \mathrm{kg} / \mathrm{min}$ respectively). Although two studies ${ }^{7}{ }^{8}$ have been conducted in this area using highly trained cyclists with similar values of $\dot{\mathrm{VO}}_{2} \mathrm{MAX}$ to those of our subjects (about 70 $\mathrm{ml} / \mathrm{kg} / \mathrm{min})$, they attained considerably lower peak power outputs $(<360 \mathrm{~W})$ during incremental tests.

SIMILAR RESPONSES IN VASTUS LATERALIS AND RECTUS FEMORIS

Based on the results of previous research with non-elite athletes, ${ }^{33-35}$ a disparity in the EMG/ exercise intensity relation would be expected to exist between the vastus lateralis and the rectus femoris. In effect, previous research has shown distinct responses in the two muscles, such as the occurrence of the EMG fatigue threshold at lower intensities in the rectus femoris. $^{34}$ This disparity could be partly attributed to kinesiological differences, as the rectus femoris (unlike the vastii muscles) is a biarticular muscle involved in both leg extension and thigh flexion. ${ }^{34}$ On the other hand, possible differences in muscle fibre composition cannot be excluded, the rectus femoris being comprised of a greater percentage of fatigable fast twitch fibres. ${ }^{33}{ }^{34}$ In this regard, few data are available on the muscle fibre composition of leg muscles in professional cyclists. In our investigation, however, $\mathrm{EMG}_{\mathrm{T} 1}$ and $\mathrm{EMG}_{\mathrm{T} 2}$ occurred at similar intensities in both muscles, suggesting a similar pattern of muscle fibre recruitment in the different leg muscles of top level cyclists as an adaptation to training and competition.

\section{CONCLUSION}

In elite cyclists the EMG activities of two leg muscles (vastus lateralis and rectus femoris) show similar patterns, with two distinct breakpoints, $\mathrm{EMG}_{\mathrm{T} 1}$ and $\mathrm{EMG}_{\mathrm{T} 2}$, occurring at an exercise intensity of $60-70 \%$ and $80-90 \%$ of $\dot{\mathrm{V}}_{2} \mathrm{MAX}$ respectively. The rms-EMG method seems to be both valid and reliable for analysing the aerobic-anaerobic transition during cycle ergometer exercise.

J L C initiated the formulation of the primary study hypothesis, discussed core ideas, designed the main part of the protocol, and contributed to the paper. A L initiated the research, coordinated the study (subject selection, test sessions, etc), and edited the manuscript. He also participated in data collection. O S participated in data collection (metabolic variables) and was responsible for analysing EMG data (detection of regression lines, statistical analysis, etc). A C also participated in EMG data statistical analysis, etc). A C also participated in EMG data analysis and data collection. We are grateful to Pedro Cuesta (Departamento de Ayuda a la Investigacion, Centro de Proceso de Datos, Complutense University of Madrid) for helping us with the use of a computer algorithm to detect multisegment linear regressions. Rosario Ureña and Margarita Pérez partially participated in data collection (blood lactate, ventilatory variables, etc) during the tests. 
1 Moritani T, deVries HA. Anaerobic threshold determination by surface electromyography. Re-examination of the
relationship between the surface integrated electromyorelationship between the surface integrated electromyogram (iEMG) and force of isometric contracti

2 Nagata A, Muro M, Moritani T, et al. Anaerobic threshold determination by blood lactate and myoelectric signals. $7 p n$ F Physiol 1981;31:585-97.

3 Viitasalo JT, Luhtanen P, Rahkila P, et al. Electromyographic activity related to aerobic and anaerobic threshold in ergometer bicycling. Acta Physiol Scand 1985;124:287-93.

4 Airaksinen $\mathrm{O}$, Remes A, Kolari OJ, et al. Real-time evaluation of the anaerobic threshold with rms-EMG of working and nonworking muscles during incremental bicycle ergometer test. Acupunture Electro 1992:17:259-71.

5 Bunc V, Hofmann P, Leitner H, et al. Verification of the heart rate threshold. Eur F Appl Physiol 1995;70:263-9.

6 Chwalbinska-Moneta J, Hänninen O, Pentilla I. RelationChips 作 during incremental exercise in endurance- and speedtrained

7 Helal JN, Guezennec CY, Goubel F. The aerobic-anaerobic transition: re-examination of the threshold concept including an electromyographic approach. Eur f Appl Physio 1987;56:643-9.

8 Glass C, Knowlton RG, Sanjabi PB, et al. The effect of exercise induced glycogen depletion on the lactate, ventilatory and electromyographic thresholds. F Sport Med Phys Fitnes 1997;37:32-40.

9 Petrofsky JS. Frequency and amplitude analysis of the EMG during exercise on a cycle ergometer. Eur f Appl Physiol 1977;41:1-15.

10 Taylor AD, Bronks R. Electromyographic correlates of the transition from aerobic to anaerobic metabolism in treadmill running. Eur $\mathcal{F}$ Appl Physiol 1994;69:508-15.

11 Rothstein L. Research design and statistics for physical education. Englewood Cliffs, NJ: Prentice-Hall, 1985.

12 Lucía A, Pardo J, Durántez A, et al. Physiological differences between elite and professional road cyclists. Int $\mathcal{F}$ Sports between elite and profe
Med 1998;19:342-8.

13 Lucía A, Vaquero AF, Pérez M, et al. Electromyographic response in cardiac transplant patients. A new method for anaerobic threshold determination? Chest 1997;111:1571-6.

14 American College of Sports Medicine. Guidelines for exercise testing and prescription. Philadelphia: Lea and Febiger, 1986:95-6.

15 Davis JA. Anaerobic threshold: a review of the concept and directions for future research. Med Sci Sports Exerc 1985;17:6-18.

16 Weltman A, Snead D, Seip R, et al. Reliability and validity of a continuous incremental treadmill protocol for the determination of lactate threshold, fixed blood lactate concentrations, and VO max. Int 7 Sports Med 1990;11:26-32.

17 Heck H, Mader A, Hess G, et al. Justification of the 4-mmol/1 lactate threshold. Int $\mathcal{F}$ Sports Med 1985;6:117-30.

18 Moritani T, Muro M. Motor unit activity and surface electromyogram power spectrum during increasing force of contraction. Eur F Appl Physiol 1987;56:260-5.
19 Green HJ, Patla AE. Maximal aerobic power: neuromuscular and metabolic considerations. Med Sci Sports Exerc 1992;24:38-46.

20 Bland JM, Altman K. Statistical methods for assessing agreement between methods of clinical measurement. Lancet 1986;1:307-10.

21 Smolander J, Kolari PJ, Korhonen O, et al. Aerobic and anaerobic responses to incremental exercise in thermoneutral and a hot environment. Acta Physiol Scand 1986;128: $15-21$.

22 Nilsson J, Tesch P, Thorstensson A. Fatigue and EMG of repeated fast voluntary contractions in man. Acta Physiol Scand 1977;101:194-8.

23 Essen B. Intramuscular substrate utilization during prolonged exercise. Ann NY Acad Sci 1977;301:30-44.

24 Takaishi T, Ono T, Yasuda Y. Relationship between muscle fatigue and oxygen uptake during cycle ergometer exercise with different ramp slope increments. Eur f Appl Physiol 1992;65:335-9.

25 Conconi F, Grazzi G, Casoni I, et al. The Conconi test: methodology after 12 years of application. Int $\mathcal{F}$ Sports Med 1996;17:509-19.

26 Favero TG, Zable AC, Coletr D, et al. Lactate inhibits $\mathrm{Ca}^{2+}$ activated $\mathrm{Ca}^{2+}$-channel activity from skeletal muscle sarcoplasmic reticulum. F Appl Physiol 1997;82:447-52.

27 Vøllestad NK, Blom PCS. Effect of varying exercise intensity on glycogen depletion in human muscle fibers. Acta Physiol Scand 1985;125:395-405.

28 Braakhekke JP, Joosten EMG, Stegeman DF. Surface EMG, McArdle's disease and exercise intolerance. Muscle Nerve 1986;9:669-70.

29 Bouissou P, Estrade PY, Goubel F, et al. Surface EMG power spectrum and intramuscular $\mathrm{pH}$ in human vastus ateralis muscle during dynamic exercise. 7 Appl Physiol 1989;67:1245-9.

30 Rowell LB, O'Learly DS. Reflex control of the circulation during exercise: chemoreflexes and mechanoreflexes. 7 Appl Physiol 1990;69:407-18.

31 Morikawa T, Ono Y, Sasaki K, et al. Afferent and cardiodynamic drives in the early phase of exercise hyperpnea in humans. f Appl Physiol 1989;67:2006-13.

32 Mateika JH, Duffin J. Coincidental changes in ventilation and electromyographic activity during consecutive incremental exercise tests. Eur f Appl Physiol 1994:68. $54-61$.

33 Miyashita M, Enehisa H, Nemoto I. EMG related to anaerobic threshold. F Sport Med Phys Fitness 1981;21:20917.

34 Housh TJ, deVries HA, Johnson GO, et al. Electromyographic fatigue thresholds of the superficial muscles of the quadriceps femoris. Eur $\mathcal{F}$ Appl Physiol 1995;71: 131-6.

35 Taylor AD, Bronks R. Effect of acute normobaric hypoxia on quadriceps integrated electromyogram and blood metabolites during incremental exercise to exhaustion. Eur f Appl Physiol 1996;73:121-9.

\section{Take home message}

During incremental cycle ergometer tests performed by top level cyclists, the relation between EMG activity (rms-EMG) of exercising muscles (vastus lateralis and rectus femoris) and exercise intensity exhibits two breakpoints, at around $60-70 \%$ and $80-90 \%$ of $\dot{\mathrm{V}}_{2} \mathrm{MAX}$ respectively. rms-EMG may be used as a complementary indicator of the aerobic-anaerobic transition in physiological evaluations of cyclists. 